\section{LHC Design Near Final}

Keith Potter, the LHC Experiments Area Cordinator, reviews the status of CERN's Large Hadron Collider project.

There has been considerable progress in the design of CERN's Large Hadron Collider (LHC), and in the LHC project as a whole, since the project was described in Europhysics News in 1992. One of the most important milestones was passed in December 1994 when the CERN Council of 19 European countries approved the project. The detailed construction schedule, however, will only be decided in 1997, when it will be known how many non-member countries have responded to the CERN Council's invitation to join the project. If all the required funds are available, the plan is to have $7 \mathrm{TeV}$ proton beams colliding in 2004; if not, it may be necessary to reprogramme in such a way as to offer lower energy collisions in 2004, and upgrade to the full energy in 2008.

L. Evans, the CERN Director responsible for construction of the collider, recently reported that the machine design has developed in a very satisfactory manner. What he believes is now close to the final design is described in a CERN report entitled The Large Hadron Collider, Conceptual Design [CERN/AC/95-05 (LHC), 20 October 1995]. This document incorporates all changes with respect to the previous version of the so-called "White Book", and will now serve as the basis for the detailed engineering design.

\section{Performance Aims Remain Unchanged}

The performance aims are almost identical to those reported in 1992, although a number of design decisions have been taken to save money and increase performance margins, offering a greater chance of reaching design performance on the planned time scale. For example, since only two experiments are now required to run simultaneously at the highest luminosity, to search for the Higgs boson, the number of bunches has been optimised and the bunch spacing increased to $25 \mathrm{~ns}$. A symmetric arrangement of these two experiments has also been chosen. This is expected to help in controlling the disturbance to the operation of the collider owing to the electromagnetic forces which an individual proton suffers each time it passes through the other beam at a collision point. This so called "beam-beam effect" is expected to be the principal

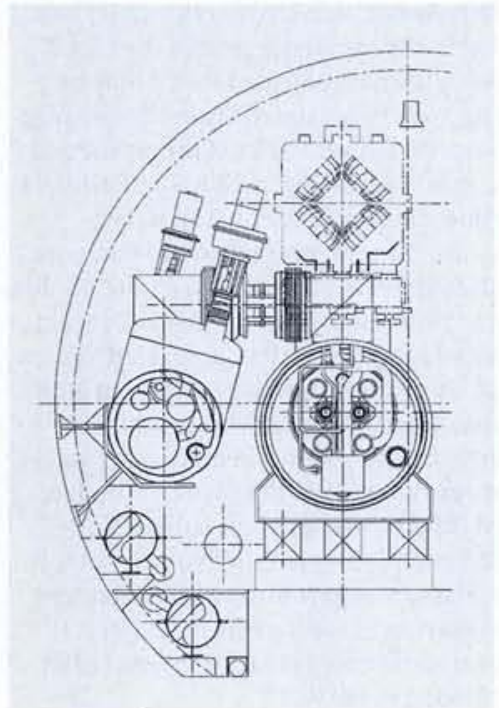

A cross-section through one-half of the machine tunnel with the twin-aperture superconducting magnet of the LHC installed below a LEP quadrupole.

The separated cryogenic feedline and connecting valve box, new developments, can be seen on the left.

\section{Main LHC parameters}

\begin{tabular}{|c|c|c|}
\hline & Protons & $\mathrm{Pb}$-lons \\
\hline Centre-of-mass total energy, $\mathrm{TeV}$ & 14 & 1148 \\
\hline Magnetic field in bending magnets, $T$ & 8.4 & 8.4 \\
\hline Initial luminosity per collision region, $\mathrm{cm}^{-2} \mathrm{~s}^{-1}$ & $10^{34}$ & $2 \times 10^{27}$ \\
\hline Number of bunches per beam & 2835 & 608 \\
\hline Bunch spacing, $\mathrm{m} / \mathrm{ns}$ & $7.5 \mid 25$ & $37.4 \mid 124.8$ \\
\hline Number of particles per bunch & $10^{11}$ & $9.4 \times 10^{7}$ \\
\hline Number of collision regions assumed & 2 & 1 \\
\hline Beta parameter at interaction point, $m$ & 0.5 & 0.5 \\
\hline r.m.s. beam radius at collision point, $\mathrm{mm}$ & 16 & 15 \\
\hline r.m.s. collision region length, $\mathrm{cm}$ & 5.4 & 5.3 \\
\hline r.m.s. energy spread, $\Delta E / E$ & $1.1 \times 10^{-4}$ & $1.1 \times 10^{-4}$ \\
\hline Beam crossing angle, mrad & 200 & $" 100$ \\
\hline Luminosity lifetime, $h$ & 10.0 & 6.7 \\
\hline Stored beam energy, MJ & 334 & 4.8 \\
\hline Synchrotron radiation (per beam), kW & 3.6 & 3.6 \\
\hline
\end{tabular}

LHC performance limitation. The design luminosity which is the proportionality factor that, multiplied by the cross-section for any process, gives the event rate, remains $10^{34} \mathrm{~cm}^{-2} \mathrm{~s}^{-1}$, but the ultimate value could be twice as high. A second, extremely important, increase in the safety margins has been achieved by redesigning the magnet lattice, in such a way as to increase the total length of twin-aperture dipoles fitted into the arcs of the LEP tunnel. For 7 $\mathrm{TeV}$ proton beams, a magnetic field of only $8.4 \mathrm{~T}$ is now needed - a value which is comfortably below those being routinely achieved in industrially produced prototypes. The $\mathrm{R} \& \mathrm{D}$ programme for the dipole magnets still has at least two years to run, but the design team is now very confident that all 1232 of the final dipoles will achieve $9 \mathrm{~T}$, providing a margin with respect to routine operation which is vital if the collider is to operate reliably with good efficiency for particle physics experiments.

\section{Mechanical Design and Cryogenics Modified}

As a result of a highly successful first "string test", in which two and later three of the first $10 \mathrm{~m}$ long dipole magnets and a prototype quadrupole were operated together in a $35-45 \mathrm{~m}$ long "string", very similar to a half-cell of the regular lattice, there have been a number of changes made to the mechanical and cryogenic layouts of the LHC. In order to avoid having to excavate new underground caverns, all cryogenic equipment has been clustered into existing structures at the even-numbered points. Systems tests on the string have shown that this will be perfectly feasible, with a separate cryogenic feed line. It had previously been thought that the most economic solution would be to integrate all feed lines into the magnet cryostat, but a separate line which now becomes possible and economic, has many obvious engineering and operational advantages. It separates the cryogenic system from the magnet system and considerably simplifies installation. However, the most visually obvious difference in the proposed tunnel layout results from the decision to place the heavy LHC cryostats on the floor of the tunnel and to reinstall the much lighter magnets of the existing LEP machine above them - an arrangement that is better adapted to a second-stage electronproton collider.

The mix of machine utilities and experiments to be installed in the eight straight sections of the LHC have not changed, but the layout has now been frozen with most of the decisions emerging during cost optimization. No experiments are yet formally approved, but the two high-luminosity general-purpose detectors 
ATLAS and CMS have been recommended for approval by the LHC experiments committee. They will be installed opposite each other in new underground areas at points $\mathrm{P}_{1}$ and $\mathrm{P}_{5}$. Two more experiments in the initial programme could be installed in the existing LEP experimental facilities at $\mathrm{P}_{2}$ and $\mathrm{P} 8$. ALICE will be a specialised experiment using heavy ions, while LHC-B will be optimised for studying the physics of the Beauty quark and $\mathrm{CP}$ violation. Only at these four points $\left(\mathrm{P}_{1}, \mathrm{P}_{2}, \mathrm{P}_{5}\right.$ and $\left.\mathrm{P} 8\right)$ will the LHC beams cross over from the inner to the outer arc, or vice versa. At the other four points, machine utilities can be installed without bringing the beams into a common vacuum pipe, thus avoiding the need for expensive bending magnets.

The straight sections around $\mathrm{P}_{3}$ and $\mathrm{P}_{7}$ will be used for the very important beam-cleaning sections, systems of collimators which will ensure that all particles which fall outside the dynamic aperture of the machine in any of the six dimensions of phase space, will be safely removed and absorbed into suitable shielding. If they were to be allowed to circulate until they struck the vacuum pipe near superconducting magnets, these particles would deposit their energy causing superconducting to normal transitions, or "quenches". The efficiency to be achieved in these cleaning sections is $99.9 \%$, as the LHC halo is expected to be fed by $10^{9}$ protons per second, while as few as $10^{6}$ may cause a quench.

It has very recently been decided to install separated RF accelerating cavities on each beam, each side of $\mathrm{P}_{4}$. This requires an increased separation of the beams in order to have enough space for the superconducting $400 \mathrm{MHz}$ cavities. Separated RF became necessary to reduce beam loading and power dissipation in the couplers to manageable values, but it will also give greater flexibility during filling and operating, and allow the LHC to collide particles or ions with different charge-to-mass ratios. An interest has already been expressed in colliding protons against lead ions. The cavity layout which has been adopted will allow the use of $\mathrm{P}_{4}$ for a future colliding beam experiment, if needed.

Injection of $450 \mathrm{GeV}$ protons from the CERN SPS ring will be immediately upstream of $\mathrm{P}_{2}$ for the clockwise rotating beam, and

A schematic layout showing the assignment of the eight long straight sections of the LHC to experiments and utilities.

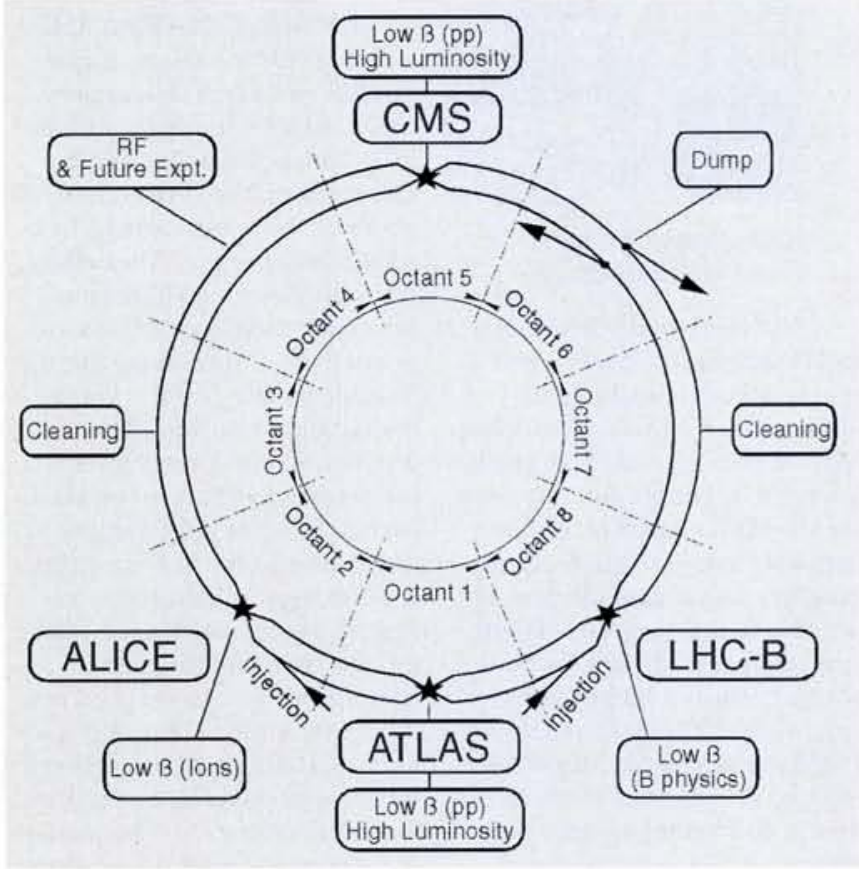

of P8 for the opposing beam. The whole chain of injectors already exists and recent beam studies have shown that the performance required is within reach. Indeed it is expected that commissioning of the LHC will profit from the availability of much lower emittance beams at lower intensities. These smaller cross-section beams are expected to allow useful luminosities of around $10^{33} \mathrm{~cm}^{-2} \mathrm{~s}^{-1}$ to be reached, even if beam intensities are initially limited operationally by magnet quenches or for any other reason.

The beams, which will have total energies of up to $334 \mathrm{MJ}$, must be safely extracted and absorbed in external beam dumps at the end of LHC colliding beam runs. The extraction system of fast kickers and septum magnets will be installed at P6 with the absorber blocks some $600 \mathrm{~m}$ away, in caverns $100 \mathrm{~m}$ underground, which will be accessed from the machine tunnel and require no new surface areas or access shafts. Details of the machine layout, with its new underground caverns, surface buildings and services are now being finalised in order to prepare an environmental impact report. The report will be presented to the relevant French and Swiss authorities in early 1996, leaving time for the necessary authorisations to be delivered and construction work to start in 1998 , as required for a 2004 start-up date.

\section{Final Guidelines}

The original version of the International Union of Pure and Applied Physics (IUPAP) Guidelines for the Major Physics Users Facilities, which were proposed by the United States Liaison Committee (USLC) in 1994, have been widely circulated. The IUPAP President received many positive responses supporting the Guidelines, as well as a few critical comments made in the context of existing large facilities that recover operating costs from users. In the light of these comments a revised Guidelines, which explicitly mention a realistic special treatment for such existing facilities, have been formulated by the USLC. They were approved by the IUPAP Executive Council in September 1995 and will be presented to the IUPAP General Assembly in Uppsala in September 1996 for ratification.

The Guidelines are the result of an extensive consultation within IUPAP, liaison committees, the UNESCO Physics Action Council, and the physics community at large. Thus, the final version, given on the next page, incorporates a wide perspective on such major facilities from many different disciplines and enjoys general support within the physics community.

IUPAP anticipates that the Guidelines, which are intended to apply to the inter-regional or international use of major facilities, will be accepted widely within the international physics community and that all future facilities will be planned, from the beginning, in conformity with the Guidelines. Furthermore, IUPAP has brought the Guidelines to the attention of other member scientific Unions of the International Council of Scientific Unions (ICSU). It expects that they will prove to be of broad interest and will eventually be generalised to become ICSU Guidelines.

In commenting on the need for common guidelines, IUPAP stresses that effective inter-regional and international cooperation is vital because scientific institutions sometimes set up restrictive political and financial goals. Guidelines benefit science by promoting open access, given that guest imbalances will tend to even out among the richer nations, while less affluent regions can contribute to the vitality of user facilities with talented scientists and individual experiments. 